\title{
EVALUACIÓN DE PENTACLORONITROBENCENO (PCNB) EN EL CONTROL DE LA PUDRICIÓN RADICULAR CAUSADA POR Rhizoctonia solani KUHN EN FRIJOL COMÚN (Phaseolus vulgaris L.) $)^{1}$
}

\author{
Sergio Pichardo Guido ${ }^{2}$
}

\section{COMPENDIO}

Este experimento fue conducido en La Compañía, Estación Experimental del Instituto Superior de Ciencias Agropecuarias (ISCA), ubicada en el departamento de Carazo, Nicaragua. Fue realizado en época de primera (junio a septiembre, 1987), con los siguientes objetivos: 1) Determinar la dosis más adecuada de $P C N B$ contra R. solani en frijol, 2) Evaluar el efecto de la mezcla de PCNB + Metalaxy1 sobre el rendimiento del frijol, 3) Determinar el porcentaje de pérdidas causadas por R. solani en frijol, 4) Seleccionar la mejor variedad de frijol en este experimento. Los resultados mostraron que la dosis de $6.81 \mathrm{~kg} / \mathrm{ha}$ de $P C N B$ fue la mejor produciendo un rendimiento de $3 \%$ más que el control y un $12 \%$ más que la mayor dosis de PCNB usada $(11.36 \mathrm{~kg} / \mathrm{ha})$. La variedad Revolución 84 produjo el más alto rendimiento.

Palabras clave adicionales: Control químico.

\section{INTRODUCCIÓN}

En Nicaragua el frijol (Phaseolus vulgaris L.) es el segundo cultivo en orden de importancia, después del maíz (Zea mays L.) Es un cultivo evidentemente susceptible a muchos factores adversos que pueden reducir considerablemente su productividad. En muchas áreas del mundo donde crece el frijol, las enfermedades son los factores más importantes de las reducciones en el rendimiento del cultivo (Pastor, 1985; Llano \& Campos, 1983).

Los investigadores han prestado atención a muchas enfermedades que atacan al cultivo. Sin embargo, las pudriciones radiculares que afectan al frijol han sido muy poco estudiadas en América Latina, en comparación a otras enfermedades que afectan al cultivo (Schwartz y Gálvez, 1980, Llano 1987). Se han observado causando considerable daño a frijoles en Brasil, Perú, México, Nicaragua, Estados Unidos y muchos otros países (Abawi, 1985). De acuerdo con Geber (1987) R.solani es un patógeno muy frecuente atacando frijol en Nicaragua. Ella encontró que en Carazo 99\% de las plantas fueron afectadas por patógenos del suelo y que $R$. solani fue el patógeno más frecuente en los aislamientos realizados. De las pudriciones radicales que afectan al frijol, la causada por $R$. solani es muy común en las áreas tropicales (Pastor, 1985). En estas áreas es responsable de considerables pérdidas en muchos cultivos, incluyendo al frijol (Lifshitz et al. 1985; Raker, 1970). R. solani ha sido encontrado en la mayoría de los suelos cultivados y no cultivados,

\begin{abstract}
${ }^{3}$
This experiment was conducted at "La Compañía", Estación Experimental del Instituto Superior de Ciencias Agropecuarias (ISCA), which is located in the department of Carazo, Nicaragua. The trial was conducted during the months of June through September, 1987 with the following objectives: 1) To determine the appropiate rate of pentachloronitrobenzene (PCNB) for control of Rhizoctonia solani in common beans, 2) To evaluate the effect of mixing PCNB and "Metalaxyl" on grain yield of common beans, 3) To determine yield reduction percentages caused by $R$. solani in common beans and 4) To select the best common bean variety in this experiment relative to the defined treatments. The results demonstrated that PCNB applied at a rate of $6.81 \mathrm{~kg} / \mathrm{ha}$ was $3 \%$ higher than the check and $12 \%$ higher than the highest rate of PCNB $(11.36 \mathrm{~kg} / \mathrm{ha})$. The variety "Revolución 84 " provided the highest yields compared to other entries.
\end{abstract}

teniendo la capacidad para atacar un amplio número de plantas taxonómicamente diferentes (Ogoshi, 1997; Tapia y García, 1983; Pastor, 1985; Schwartz y Gálvez, 1980; Oda, 1972; Baker, 1970).

Los aislamientos de $R$. solani difieren en patogenicidad y morfología, así como en características culturales y fisiológicas (Ogoshi, 1987). No es una especie única, sino una colección de poblaciones no intercruzables (Anderson, 1982), que da origen al concepto de grupo anastomosis (AG) en el cual se agrupa a organismos de la misma especie que pueden entrecruzar sus hifas, pero no a aquellos que son genéticamente diferentes. Anastomosis hifal ocurre en aislamientos de campo del mismo grupo, pero no en aislamientos de diferentes grupos (Anderson, 1977, Andersen 1982; Ogoshi, 1987).

De los AG reportados por Parmeter (Parmeter et al, 1969) el AG 4 es el que ataca frijol y casi todas las especies angiospermas (Anderson, 1982). R. Solani puede inducir damping-off, cáncer del tallo, pudrición de la raíz, pudrición de la vaina (Schwarts y Gálvez, 1980). De acuerdo a Pastor (1985) la temperatura óptima para el hongo cuando se encuentra formando chancros en frijol es de $18^{\circ} \mathrm{C}$. El daño es usualmente más severo

1 Presentado en la XXXV Reunión Anual del PCCMCA, San Pedro Sula, Honduras, 1989.

2 Técnico Plant Science Program ISCA-SLU. Nicaragua.

3 El Abstract ts traducción del compendio. Elaborado por el Comité Editorial para mostrar el formato de presentación de los artículos.

Publicado en Agronomía Mesoamericana Vol. 1 (1990). 
durante las primeras dos o tres semanas después de la siembra (StockweIl y Hanchey, 1987; Geber, 1987). La susceptibilidad de las plantas al ataque por parte de $R$. solani declina con la maduración y lignificación de los tejidos al tiempo que las plantas van creciendo (Baker, 1970; Pastor, 1985).

El control químico sólo es efectivo durante la germinación y el desarrollo inicial de las plantas (Pastor, 1985). Pentacloronitrobenceno (FNCB) ha sido reportado por muchos investigadores como el fungicida más comúnmente usado para el control de $R$. solani (Bristow et al, 1973; Ko y Oda, 1972; Rodríguez-Kabana, 1980). Es específico y selectivo controlando $R$. solani en el cual causa una fuerte supresión de su crecimiento, más que su destrucción (Bristow et al, 1973; Ko y Oda, 1972).

Sin embargo, hay otros patógenos como Pythium aphanidermatum, P. ultimun, Phytophthora y Fusarium que no son controlados por este fungicida (Katan y Lockwood, 1970; Rodríguez-Kabana, 1980; Dekker y Georgopoulos, 1982). Sin embargo, según Geber (comunicación personal) el PCNB fue usado en Suecia para el control de Fusarium, antes de que su uso fuera prohibido en ese país, debido a sus propiedades carcinogénicas. Estos hongos que no son afectados por el fungicida mas, los cuales pueden evitarse con el uso de fungicidas menos selectivos o una combinación de fungicidas efectiva contra diferentes organismos (Leach y Gerber, 1970). Uno de los fungicidas que puede ser usado para controlar Pythium es Metalaxyl, el cual fue usado anteriormente (Llano, 1987), logrando incrementar el rendimiento en $38 \%$. Es un fungicida altamente específico contra Domycetes (Dekker y Georgopoulos, 1982; Worthing, 1979; Cardoso, 1987).

En base a la problemática descrita, el trabajo fue realizado persiguiendo los siguientes objetivos: Determinar la dosis más adecuada de PCNB contra $R$. solani en frijol. Evaluar el efecto de la mezcla de PCNB + Metalaxyl sobre el rendimiento del frijol. Determinar el porcentaje de pérdidas causadas por $R$. solani en frijol, y seleccionar la mejor variedad de frijol en este experimento.

\section{MATERIALES Y METODOS}

Este experimento fue conducido en La Compañía, Estación Experimental del Instituto Superior de Ciencias Agropecuarias (ISCA), ubicada en el departamento de Carazo, Nicaragua. Fue realizado en siembras de Primera (Junio - septiembre, 1987). El tipo de suelo es franco-arenoso con buen drenaje y situado sobre una ligera pendiente. La tierra fue preparada convencionalmente con un pase de arado y dos pases de grada.

La fertilización fue hecha con urea $46 \% \mathrm{~N}$ a dosis de $59.5 \mathrm{~kg} / \mathrm{ha}$ y completo (10-30-10) a $90.9 \mathrm{~kg} / \mathrm{ha}$. Los fertilizantes fueron aplicados al fondo del surco antes de colocar las semillas, las cuales fueron seleccionadas y contadas para obtener una población de 250,000 plan- tas /ha. Benorny1 y Deltametrina fueron aplicados para controlar algunas enfermedades y plagas del follaje. El control de malezas fue realizado manualmente y también haciendo uso de Paraquat a dosis comercial, rociado en la calle de los surcos.

Los fungicidas usados en el experimento: PCNB y Metalaxyl, fueron rociados sobre las semillas en el fondo del surco antes de ser tapadas. Las variedades usadas fueron tres rojas: Rojo Nacional, Revolución 79, Revolución 84; y dos variedades negras: lca Pijao y Negro Huasteco.

El experimento fue arreglado como parcelas divididas en bloques completamente al azar (BCA), con cinco tratamientos y cinco repeticiones. Los fungicidas (Cuadro 1), fueron aplicados en la parcela principal (30 surcos/8 m de largo) y las variedades fueron plantadas en cada subparcela (6 surcos/variedad). La distancia entre surcos fue de $0.5 \mathrm{~m}$. El área efectiva del ensayo fue de $3,000 \mathrm{~m}^{2}$.

Cuadro 1. Dosis de PCNB y Metalaxyl

\begin{tabular}{cccc}
\hline No & Dosis (kg/ha) & Fungicida & Observaciones \\
\hline 1 & 0.00 & PCNB & \\
2 & 3.63 & PCNB & \\
3 & 6.81 & PCNB & \\
4 & 6.81 & PCNI3 + Metalaxyl & Mezcla \\
5 & 11.36 & PCNB & \\
\hline
\end{tabular}

Se hicieron 4 muestreos a los 10, 20, 40 y 60 días después de la siembra. En el primero y segundo muestreo se registró severidad e incidencia de la enfermedad, nodulación, desarrollo radicular y peso seco de la parte verde y las raíces por separado, y en el tercero y cuarto muestreo se registró el peso fresco y peso seco de las plantas.

Se usaron escalas subjetivas para registrar nodulación y desarrollo radicular y una escala del CIAT para registrar la severidad de los chancros. Se efectuaron 4 muestreos en total, dos en cada borde opuesto de la parcela útil.

1 En el primer muestreo, se tomaron 10 plantas en un área de borde a los 10 días después de siembra, las cuales fueron arrancadas cuidadosamente.

2 En el segundo muestreo, a los 20 días fueron tomadas 5 plantas del borde opuesto que en el primer muestreo.

3 En el tercero y cuarto muestreo se tomó únicamente 5 plantas por subparcela. En el cuarto muestreo sólo se usaron 2 bloques para la obtención del peso seco.

En todos los casos las muestras fueron mantenidas en un horno eléctrico por 3 días a temperatura de $105^{\circ} \mathrm{C}$.

Para rendimiento fueron cosechados los cuatro surcos centrales, las vainas fueron separadas de los granos, 
se pesó, el contenido de agua fue medido y los valores de rendimiento corregidos a $14 \%$ de humedad.

\section{RESULTADOS}

1. Severidad de la enfermedad. En la primera lectura a los 10 días después de siembra (dds) la severidad de la enfermedad fue disminuyendo con el aumento del nivel de fungicida aplicado, exceptuando en la dosis de $3.63 \mathrm{~kg} / \mathrm{ha}$ y este efecto se mantuvo en la segunda lectura a los 20 dds (cuadro 2), donde además, se produjeron diferencias estadísticas, siendo el control diferente de la 4a. y la 5a. dosis de PCNB.

La respuesta varietal fue poco intensa en la primera lectura, pero su intensidad aumentó en la segunda lectura, donde el testigo Rojo Nacional fue superado ampliamente por el resto de variedades probadas. No se encontraron diferencias estadísticas (Cuadro 2).

2. Incidencia de la enfermedad. Hubo un efecto temprano más fuerte con PCNB donde la incidencia de la enfermedad fue disminuyendo con el aumento de la dosis $\mathrm{M}$ fungicida. Diferencias estadísticas fueron observadas entre el control y el resto de tratamientos, los cuales

Cuadro 2. Severidad de la enfermedad

\begin{tabular}{|c|c|c|c|c|c|}
\hline $\begin{array}{c}\text { Nivel PCNB } \\
\text { (kg/ha) }\end{array}$ & $\begin{array}{c}\text { Lectura } 1 \\
(\%)\end{array}$ & $\begin{array}{c}\text { Lectura } 2 \\
(\%)\end{array}$ & Variedad L & $\begin{array}{l}\text { Lectura } \\
\qquad(\%)\end{array}$ & $\begin{array}{c}1 \text { Lectura } 2 \\
(\%)\end{array}$ \\
\hline 0.00 & 100 & $100 \mathrm{~b}$ & R. Nacional & 100 & 100 \\
\hline 3.63 & 104 & $92 \mathrm{ab}$ & Rev. 79 & 89 & 115 \\
\hline 6.81 & 87 & $85 \mathrm{ab}$ & Rev. 84 & 115 & 117 \\
\hline $6.81+3^{*}$ & 76 & 79 a & Negro Huast. & t. 94 & 114 \\
\hline 11.36 & 75 & $78 \mathrm{a}$ & Ica Pijao & 95 & 117 \\
\hline $\begin{array}{l}{ }^{*} \text { Mezcla de } P \\
\text { En lectura 1: } \\
\text { En lectura 2: }\end{array}$ & $\begin{array}{l}\mathrm{PCNB}+\mathrm{M} \\
100=3.64 \\
100=4.20\end{array}$ & axy1 & $\begin{array}{l}\text { R. Nacional es } \\
\text { En lectura 1: } \\
\text { En lectura 2: }\end{array}$ & $\begin{array}{l}\text { es usado } \\
100=3 . \\
100=3 .\end{array}$ & $\begin{array}{l}\text { como te } \\
49 \\
24\end{array}$ \\
\hline
\end{tabular}

Lecturas con la misma letra indican que no hay diferencias estadísticas con Tukey $5 \%$. también difirieron entre ellos. La 2a. y la 3a. fueron diferentes de la 4a. dosis. El efecto continuó después de los 20 dds y se mantuvo con los niveles del fungicida exceptuando en la mezcla usada (Cuadro 3). También en esta segunda lectura hubo diferencias estadísticas, pero en este caso el control sólo difirió de la mayor dosis de fungicida aplicada. En general las variedades responden genéticamente. Las mejoradas sufrieron menor ataque, especialmente en las variedades negras. El efecto en las variedades disminuyó con el tiempo, y no se observaron diferencias estadísticas (Cuadro 3).

3. Peso seco de raíces. En la primera lectura no hubo efecto del PCNB sobre el peso de las raíces, exceptuando la dosis de $11.36 \mathrm{~kg} / \mathrm{ha}$ donde el peso seco de las raíces disminuyó en un $6 \%$. Sin embargo, en la segunda lectura realizada a los $20 \mathrm{dds}$, el efecto del fungicida disminuyó el peso seco de las raíces, llegando a producirse una merma de $16 \%$ en la mayor dosis de PCNB. Las variedades rojas introducidas, al igual que las negras respondieron positivamente en esta variable, superando claramente a la variedad testigo en ambas lecturas. No se observaron diferencias estadísticas, tanto para dosis como para variedades (Cuadro 4).

Cuadro 3. Incidencia de la enfermedad

\begin{tabular}{|c|c|c|c|c|c|}
\hline $\begin{array}{c}\text { Nivel PCNB } \\
\text { (kg/ha) }\end{array}$ & $\begin{array}{l}\text { Lectura } \\
(\%)\end{array}$ & $\begin{array}{c}1 \text { Lectura } 2 \\
(\%)\end{array}$ & 2 Variedad & $\begin{array}{c}\text { Lectura } \\
(\%)\end{array}$ & $\begin{array}{c}1 \text { Lectura } 2 \\
(\%)\end{array}$ \\
\hline 0.00 & $100 \mathrm{c}$ & $100 \mathrm{~b}$ & R. Nacional & 100 & 100 \\
\hline 3.63 & $58 \mathrm{~b}$ & $84 \mathrm{ab}$ & Rev. 79 & 100 & 92 \\
\hline 6.81 & $51 \mathrm{~b}$ & $82 a b$ & Rev. 84 & 105 & 100 \\
\hline $6.81+3^{*}$ & $24 \mathrm{a}$ & $90 \mathrm{~b}$ & Negro Huast. & 75 & 87 \\
\hline 11.36 & $25 \mathrm{a}$ & $66 \mathrm{a}$ & Ica Pijao & 77 & 89 \\
\hline $\begin{array}{l}\text { * Mezcla de } \\
\text { En lectura 1: } \\
\text { En lectura 2: }\end{array}$ & $\begin{array}{l}P C N B+N \\
100=59 \\
100=82\end{array}$ & $\begin{array}{ll}\text { alaxyl } & F \\
& \mathrm{E} \\
& \mathrm{E}\end{array}$ & $\begin{array}{l}\text { R. Nacional es } \\
\text { En lectura 1: } 1 \\
\text { En lectura 2: } 1\end{array}$ & $\begin{array}{l}\text { es usado c } \\
100=33 \\
100=74\end{array}$ & no testigo \\
\hline
\end{tabular}

Lecturas con la misma letra indican que no hay diferencias estadísticas con Tukey $5 \%$.

Cuadro 5. Peso seco de plantas (sin raíces)

\begin{tabular}{|c|c|c|c|c|c|c|c|c|c|}
\hline $\begin{array}{c}\text { Nivel PCNB } \\
\text { (kg/ha) }\end{array}$ & $\begin{array}{c}\text { Lect. } 1 \\
(\%)\end{array}$ & $\begin{array}{c}\text { Lect. } 2 \\
(\%)\end{array}$ & $\begin{array}{c}\text { Lect. } 3 \\
(\%)\end{array}$ & $\begin{array}{c}\text { Lect. } 4 \\
(\%)\end{array}$ & Variedad & $\begin{array}{c}\text { Lect. } 1 \\
(\%)\end{array}$ & $\begin{array}{c}\text { Lect } 2 \\
(\%)\end{array}$ & $\begin{array}{c}\text { Lect. } 3 \\
(\%)\end{array}$ & $\begin{array}{c}\text { Lect. } 4 \\
(\%)\end{array}$ \\
\hline 0.00 & $100 \mathrm{~b}$ & $100 \mathrm{c}$ & $100 \mathrm{~b}$ & 100 & R. Nac. & 100 & 100 & 100 & $100 \mathrm{a}$ \\
\hline 3.63 & $89 \mathrm{ab}$ & $94 \mathrm{bc}$ & $82 \mathrm{ab}$ & 101 & Rev. 79 & & 117 & 99 & $11694 \mathrm{a}$ \\
\hline 6.81 & $88 \mathrm{ab}$ & $83 a b$ & $81 \mathrm{ab}$ & 79 & Rev. 84 & 116 & 104 & 124 & $115 \mathrm{ab}$ \\
\hline $6.81+3^{*}$ & $99 \mathrm{ab}$ & 90 be & $90 \mathrm{ab}$ & 81 & N. Huast. & 116 & 104 & 124 & $115 \mathrm{ab}$ \\
\hline 11.36 & $85 \mathrm{a}$ & $73 a$ & $78 \mathrm{a}$ & 93 & lea Pijao & 100 & 104 & 130 & $121 \mathrm{ab}$ \\
\hline
\end{tabular}

* Mezcla de PCNB + Metalaxy1

R. Nacional es usado corno testigo

En lectura 1: $100=1.94 \mathrm{~g}$

En lectura 1: $100=1.67 \mathrm{~g}$

En lectura 2: $1005.43 \mathrm{~g}$

En lectura 2: $100=4.66 \mathrm{~g}$

En lectura 3: $10061 \mathrm{~g}$

Enlectura3: $100=44 \mathrm{~g}$

En lectura 4: $100105 \mathrm{~g}$

En lectura 4: $100=85 \mathrm{~g}$

Lecturas con la misma letra indican que no hay diferencias estadísticas $\mathrm{cm}$ Tukey $5 \%$. 
Cuadro 4. Peso seco de raíces.

\begin{tabular}{cccccc}
\hline $\begin{array}{c}\text { Nivel PCNB Lectura } \\
(\mathbf{k g} / \mathbf{h a})\end{array}$ & $\mathbf{( \% )}$ & $\begin{array}{c}\text { Lectura } 2 \\
(\boldsymbol{\%})\end{array}$ & Variedad & $\begin{array}{c}\text { Lectura } 1 \\
\mathbf{( \% )}\end{array}$ & $\begin{array}{c}\text { Lectura } 2 \\
\mathbf{( \% )}\end{array}$ \\
\hline 0.00 & 100 & $100 \mathrm{~b}$ & R. Nacional & 100 & 100 \\
3.63 & 100 & $96 \mathrm{ab}$ & Rey. 79 & 121 & 113 \\
6.81 & 100 & $89 \mathrm{ab}$ & Rey. 84 & lis & 111 \\
$6.81+3^{*}$ & 106 & $93 \mathrm{ab}$ & Negro Huast & 118 & 117 \\
11.36 & 94 & $84 \mathrm{ab}$ & Ica Pijao & 121 & 117 \\
\hline
\end{tabular}

Mezcla de PCNB + Metalaxyl R. Nacional es usado como testigo

En lectura 1; $100=59 \quad$ En lectura 1: $100=33$

En lectura 2: $100=82 \quad$ En lectura 2: $100=74$

Lecturas con la misma letra indican que no hay diferencias estadísticas con Tukey $5 \%$.

4. Peso seco de plantas. Hubo una considerable reducción del peso seco de las plantas con el uso de PCNB, mostrándose con mayor frecuencia es te fenómeno con la dosis de $11.36 \mathrm{~kg} / \mathrm{ha}$. Diferencias estadísticas para dosis se mostraron en los primeros tres de los cuatro muestreos realizados. En el lo. y 3er. muestreos el control fue diferente de la mayor dosis de PCNB usada en el trabajo, mientras que en el 2o. muestreo el control difirió de las dosis de 6.81 y de la $11.36 \mathrm{~kg} / \mathrm{ha}$. Las variedades mostraron respuesta positiva a esta variable y en la medida que pasaba el tiempo todas superaron con mayor porcentaje a la variedad testigo Rojo Nacional, pero sólo en la 4a. Lectura Revolución 84 difirió estadísticamente de la variedad testigo (Cuadro 5).

5. Nodulación. Hubo una drástica reducción de la nodulaci6n en el primer muestreo en comparación con el control. El efecto del fungicida tiende a disminuir al avanzar el tiempo y la nodulaci6n recupera aunque no se iguala con el control en ningún tratamiento. Las variedades incrementaron su producción de nódulos entre 45 a $55 \%$ en relación al testigo. Sin embargo, para la segunda lectura se mostró una drástica disminución en la nodulación en comparación con la variedad testigo, contrastando además, con el comportamiento de la formación de nódulos en la lectura efectuada a los 10 dds. Sin embargo, en ambas lecturas no hubo diferencias estadísticas, tanto para dosis como para variedades (Cuadro 6).

Cuadro 6. Nodulación

\begin{tabular}{|c|c|c|c|c|c|}
\hline $\begin{array}{c}\text { Nivel PCNB } \\
\text { (kg/ha) }\end{array}$ & $\begin{array}{c}\text { Lectura } \\
(\%)\end{array}$ & $\begin{array}{c}1 \text { Lectura } 2 \\
(\%)\end{array}$ & 2 Variedad & $\begin{array}{c}\text { Lectura } \\
(\%)\end{array}$ & $\begin{array}{c}1 \text { Lectura } 2 \\
(\%)\end{array}$ \\
\hline 0.00 & $100 \mathrm{a}$ & 100 & R. Nacional & 100 & 100 \\
\hline 3.63 & $55 \mathrm{a}$ & 97 & Rev. 79 & 155 & 91 \\
\hline 6.81 & $38 \mathrm{a}$ & 97 & Rev. 84 & 111 & 93 \\
\hline $6.81+3^{*}$ & $54 \mathrm{ab}$ & 89 & Negro Huast. & 145 & 96 \\
\hline 11.36 & $54 \mathrm{ab}$ & 94 & Ica Pijao & 145 & 97 \\
\hline
\end{tabular}

* Mezcla de PCNB + Metalaxy1 R. Nacional es usado como testigo

En lectura 1: $100=0.96 \quad$ En lectura 1: $100=0.44$

En lectura 2: $100=1.44 \quad$ En lectura 2: $100=1,44$

Lecturas con la misma letra indican que no hay diferencias estadísticas con Tukey $5 \%$.
6. Desarrollo radicular. El desarrollo radicular sufrió incremento en las dosis de PCNB, exceptuando en el tercer tratamiento, el cual fue estadísticamente diferente del control. En la segunda lectura el desarrollo radicular disminuyó con el uso del fungicida en relación al control, pero éste sólo difirió de las dosis de 6.81 y 11.36 $\mathrm{kg} / \mathrm{ha}$. Las variedades incrementaron su desarrollo radicular con respecto al testigo en la primera lectura. Sin embargo, en el segundo muestreo, aunque la variedad testigo fue superada por las otras variedades en su desarrollo radicular, este incremento fue menor en comparación con el obtenido en el primer muestreo (Cuadro 7).

7. Peso fresco de las plantas. El peso fresco fue afectado en las plantas tratadas con PCNB, tanto en la primera como en la segunda lectura, pero la dosis mayor del fungicida $(11.36 \mathrm{~kg} / \mathrm{ha})$ fue el tratamiento que más afectó el peso fresco de las plantas, siendo el único que difirió estadísticamente del control en la segunda lectura. Las variedades mejoradas mostraron un fuerte incremento de peso fresco con respecto al testigo (R. Nacional) en ambos muestreos, especialmente en las variedades negras, pero no se mostraron diferencias estadísticas entre ellas (Cuadro 8).

8. Plantas cosechadas por subparcela. El número de plantas cosechadas por subparcela no difirió estadísticamente tanto para dosis de PCNB como para variedades

Cuadro 7. Desarrollo radicular

\begin{tabular}{cccccc}
\hline $\begin{array}{c}\text { Nivel-PCNB Lectura } 1 \\
(\mathbf{k g} / \mathbf{h a})\end{array}$ & $\begin{array}{c}\text { Lectura } 2 \\
(\boldsymbol{\%})\end{array}$ & $\begin{array}{c}\text { Variedad } \\
(\mathbf{\%})\end{array}$ & $\begin{array}{c}\text { Lectura } 1 \text { Lectura 2 } \\
(\boldsymbol{\%})\end{array}$ & $\mathbf{( \% )}$ \\
\hline 0.00 & $100 \mathrm{ab}$ & $100 \mathrm{c}$ & R.Nacional & 100 & 100 \\
3.63 & $107 \mathrm{ab}$ & $101 \mathrm{c}$ & Rey. 79 & 121 & 110 \\
6.81 & $88 \mathrm{a}$ & $86 \mathrm{ab}$ & Rey. 84 & 132 & 106 \\
$6.81+3^{*}$ & $118 \mathrm{~b}$ & $97 \mathrm{bc}$ & Negro Huast. & 124 & 110 \\
11.36 & $107 \mathrm{ab}$ & $82 \mathrm{a}$ & Ica Pijao & 129 & 108 \\
\hline
\end{tabular}

* Mezcla de PCNB + Metalaxy1 R. Nacional es usado como testigo En lectura 1: $100=1.96 \quad$ En lectura 1: $100=1.68$

En lectura 2: $100=2-84 \quad$ En lectura 2: $100=2.48$

Lecturas con la misma letra indican que no hay diferencias estadísticas con Tukey 5\%.

Cuadro 8. Peso fresco de las plantas

\begin{tabular}{|c|c|c|c|c|c|}
\hline $\begin{array}{l}\text { Nivel-PCNB } \\
\text { (kg/ha) }\end{array}$ & $\begin{array}{c}\text { Lectura } 1 \\
\quad(\%)\end{array}$ & $\begin{array}{c}1 \text { Lectura } 2 \\
(\%)\end{array}$ & 2 Variedad I & $\begin{array}{c}\text { Lectura } \\
(\%)\end{array}$ & $\begin{array}{c}1 \text { Lectura } 2 \\
(\%)\end{array}$ \\
\hline 0.00 & $100 \mathrm{~b}$ & 100 & R. Nacional & 100 & $100 a b$ \\
\hline 3.63 & $88 \mathrm{ab}$ & 91 & Rey. 79 & 105 & $94 a$ \\
\hline 6.81 & $79 \mathrm{ab}$ & 76 & Rey. 84 & 115 & 135 he \\
\hline $6.81+3^{*}$ & $89 a b$ & 84 & Negro Huast. & 118 & $136 \mathrm{bc}$ \\
\hline 11.36 & $74 \mathrm{a}$ & 75 & lea Pijao & 117 & $145 \mathrm{c}$ \\
\hline $\begin{array}{l}\text { En lectura 1: } \\
\text { En lectura 2: }\end{array}$ & $\begin{array}{l}100=473 \\
100=436\end{array}$ & $\begin{array}{ll}\text { laxy1 } & \mathrm{I} \\
\mathrm{E} & \mathrm{E} \\
\mathrm{E} & \end{array}$ & $\begin{array}{l}\text { R. Nacional es } \\
\text { En lectura 1: } 1 \\
\text { En lectura 2: } 1\end{array}$ & $\begin{array}{l}100=367 \\
100=44\end{array}$ & $g$ \\
\hline
\end{tabular}

Lecturas con la misma letra indican que no hay diferencias estadísticas con Tukey $5 \%$. 
de frijol. Sin embargo, el testigo superó ligeramente al resto de los tratamientos, mientras que la mayor dosis de PCNB $11.36 \mathrm{~kg} / \mathrm{ha}$ dio el menor valor, el cual fue inferior en un 7\% con respecto al control. En las variedades sólo Revolución 84 superó en un $8 \%$ a la variedad nativa Rojo Nacional, pero ésta además, superó ligeramente al resto de las variedades probadas (Cuadro 9).

Cuadro 9. Plantas cosechadas por subparcela

\begin{tabular}{ccclcr}
\hline $\begin{array}{c}\text { Dosis } \\
\text { PCNB } \\
\text { (kg/ha) }\end{array}$ & $\begin{array}{c}\text { Promedio } \\
\text { X }\end{array}$ & $\%$ & $\begin{array}{l}\text { Promedio } \\
\text { Variedad }\end{array}$ & $\mathbf{X}$ & $\%$ \\
\hline 0.00 & $159 \mathrm{a}$ & 100 & Rojo Nac. & $153 \mathrm{a}$ & 100 \\
3.63 & $156 \mathrm{a}$ & 98 & Rev. 79 & $151^{*}$ & 99 \\
6.81 & $154^{*}$ & 97 & Rev. 84 & $166 \mathrm{a}$ & 109 \\
$* 6.81+3$ & 158 & 99 & Negro Huast. 152a & 99 \\
11.36 & $148 \mathrm{a}$ & 93 & lca Pijao & $152 \mathrm{a}$ & 99 \\
\hline
\end{tabular}

Separación de medias usando Tukey $5 \%$

* Mezcla de PCNB + Metalaxy1

Rojo Nac. es usado como testigo

Lecturas con la misma letra indican que no hay diferencias estadísticas con Tukey $5 \%$.

C.V. $=19.13$

9. Peso de 1,000 semillas. El PCNB no afectó el peso de 1,000 semillas. El análisis estadístico no mostró diferencias estadísticas para las dosis de PCNB y no hubo diferencias entre las variaciones de peso expresadas en porcentaje. Las variedades sí presentaron diferencias estadísticas. La variedad nativa Rojo Nacional sólo fue ligeramente superior a Revolución 79, y fue estadísticamente diferente de] resto de variedades por las cuales fue superada en esta variable. Las variedades de color negro en este ensayo tuvieron mayor peso que las de color rojo (Cuadro 10).

10. Rendimiento. El rendimiento no difirió estadísticamente entre lu dosis de PCNB. Sin embargo, la dosis más alta de $11.36 \mathrm{~kg} / \mathrm{ha}$ produjo el menor rendimiento, mientras que la dosis de $6.81 \mathrm{~kg} / \mathrm{ha}$ produjo el más al lo rendimiento, pero éste fue únicamente $3 \%$ ma-

Cuadro 10. Peso de 1,000 semillas (grs).

\begin{tabular}{ccrllr}
\hline $\begin{array}{c}\text { Dosis } \\
\text { PCNB } \\
\text { (kg/ha) }\end{array}$ & $\begin{array}{c}\text { Promedio } \\
\mathbf{X}\end{array}$ & $\%$ & $\begin{array}{l}\text { Promedio } \\
\text { Variedad }\end{array}$ & $\mathbf{X}$ & $\%$ \\
\hline & & & & & \\
\hline 0.00 & $146 \mathrm{a}$ & 100 & Rojo Nac. & $141 \mathrm{a}$ & 100 \\
3.63 & $145 \mathrm{a}$ & 99 & Rey. 79 & $131 \mathrm{a}$ & 93 \\
6.81 & $146 \mathrm{a}$ & 100 & Rey. 84 & $151 \mathrm{~b}$ & 107 \\
$* 6.81+3$ & $145 \mathrm{a}$ & 99 & Negro Huast. $155 \mathrm{~b}$ & 110 \\
11.36 & $149 \mathrm{a}$ & 101 & Ica Pijao & $151 \mathrm{~b}$ & 108 \\
\hline
\end{tabular}

Separación de medias usando Tukey 5\% *

Mezcla de PCNB +,Metalaxyl

Rojo Nac. es usado como testigo

Lecturas con la misma letra indican que no hay diferencias estadísticas con Tukey $5 \%$.

C.V. $=5.12$ yor que el testigo y $12 \%$ mayor que la dosis más alta del fungicida. La mezcla de PCNB + Metalaxy1 no incrementó el rendimiento del frijol y sólo superó el rendimiento obtenido en el tratamiento con $11.36 \mathrm{~kg} / \mathrm{ha}$. Las variedades sí mostraron diferencias estadísticas entre ellas en el rendimiento producido. La variedad criolla Rojo Nacional fue superada por todas las variedades restantes, y sólo fue igual estadísticamente a Ica Pijao, pero diferente estadísticamente de las demás. Revolución 84 que fue la variedad de mayor rendimiento superó en un $32 \%$ a la variedad nativa Rojo Nacional, que fue usada como testigo (Cuadro 11).

Cuadro 11. Rendimiento (kg/ha)

\begin{tabular}{ccrlrl}
\hline $\begin{array}{c}\text { Dosis } \\
\text { PCNB } \\
\text { (kg/ha) }\end{array}$ & $\begin{array}{c}\text { Promedio } \\
\text { X }\end{array}$ & $\%$ & $\begin{array}{l}\text { Promedio } \\
\text { Variedad }\end{array}$ & $\mathbf{X}$ & $\%$ \\
\hline 0.00 & $1158 \mathrm{a}$ & 100 & Rojo Nac. & $957 \mathrm{a}$ & 100 \\
3.63 & $1136 \mathrm{a}$ & 98 & Rev. 79 & $1151 \mathrm{~b}$ & 121 \\
6.81 & $1197 \mathrm{a}$ & 103 & Rey. 84 & $1261 \mathrm{~b}$ & 132 \\
$* 681+3$ & $1130 \mathrm{a}$ & 97 & Negro Huast. & $1185 \mathrm{~b}$ & 124 \\
11.36 & $1055 \mathrm{a}$ & 91 & Ica Pijao & $1112 \mathrm{ab}$ & 116 \\
\hline
\end{tabular}

Separación de medias usando Tukey $5 \%$

* Mezcla de PCNB + Metalaxyl

Rojo Nac. es usado como testigo

Lecturas con la misma letra indican que no hay diferencias estadísticas $\mathrm{cm}$ Tukey $5 \%$.

C.V. $=19.70$

\section{DISCUSIÓN}

Los resultados mostraron que la severidad e incidencia de la enfermedad disminuyó con el incremento de la dosis de PCNB. Esto concordó con Bristow et al (1973), quienes encontraron que la severidad de la enfermedad en plantas conteniendo PCNB fue significativamente menor que en plantas no expuestas al fungicida. Ellos también encontraron que el PCNB absorbido redujo el tamaño promedio de lesiones. Sin embargo, la severidad e incidencia de la enfermedad fueron mayores a los 20 días que a los 10 días después de la siembra, posiblemente debido a que el control químico de la enfermedad es solamente efectivo durante el período de germinación y desarrollo inicial de las plantas (Pastor, 1985; Schwartz y Gálvez, 1980). Además, de acuerdo con Bristow et al (1973), la concentración del PCNB en las raíces e hipocótilos de plántulas de frijol disminuye rápidamente después de la emergencia, permitiendo que el patógeno se desarrolle y cause daño a las plántulas. La más baja severidad de la enfermedad a los 10 días después de siembra también puede ser influenciada por la efectividad del método de aplicación, al rociar las semillas en el surco antes que fueran cubiertas (Shlevin y Katan, 1975). E1los obtuvieron efectivo control de Rhizoctonia solani usando PCNB cuando aplicaron el 
fungicida de esta manera. También Pastor (1985) y Moore y Conover (1954) reportaron la efectividad del método.

El peso seco de las plantas a los 10, 20 y 40 días después de la siembra y el peso de las raíces, la nodulación y el desarrollo radicular a los 10 y 20 días después de siembra, generalmente fueron menores en las plantas tratadas con PCNB y especialmente con la dosis más alta. Esto posiblemente, se deba a cierta fitotoxicidad inducida por el fungicida en las plantas que afecta su crecimiento, desarrollo y producción. Además, puede afectar la actividad microbiana en el suelo donde se encuentran las bacterias de la nodulación, ya que cualquier pesticida que se pone en contacto con el suelo de campo rápidamente induce al menos un cambio temporal en el comportamiento microbial del suelo. La alteración del equilibrio microbial por pesticidas en el suelo ha sido claramente demostrada con PCNB (Rodríguez-Kabana y Curl, 1980.

La poca actividad de Rhizoctonia solani en la siembra de primera, posiblemente se deba a la baja concentración del patógeno al comienzo de la estación lluviosa. De acuerdo con Weber (1939) el hongo sobrevive sobre restos orgánicos, pero éstos son descompuestos rápidamente en las condiciones tropicales.

El peso fresco de las plantas tuvo casi la misma tendencia que el peso seco de las mismas. Se presentaron ligeras variaciones que pueden deberse a las diferentes concentraciones de agua que tienen las distintas variedades del cultivo. Por lo tanto puede decirse que no es necesario registrar el peso fresco de las muestras tomadas, sino el peso seco debido a que éste es más representativo dado que muestra la cantidad de materia seca formada por la planta.

El número de plantas cosechadas no difirió estadísticamente, tanto para dosis como para variedades, lo mismo que en el rendimiento obtenido. Esto concuerda con lo mostrado por Bcebe et al (1981), quienes reportaron que las parcelas útiles de frijol seco fueron afectadas solamente cuando hubo una considerable reducción de las plantas cosechadas. En el rendimiento únicamente se mostraron diferencias estadísticas para las variedades de frijol, lo cual puede deberse a la diferente capacidad productiva de las distintas variedades usadas en el experimento. Revolución 84 fue la variedad que produjo el mayor rendimiento, las variedades mejoradas incrementaron su rendimiento 16 - 32\% en relación al testigo. El más bajo rendimiento fue obtenido con la dosis más alta de] fungicida, donde la severidad e incidencia de la enfermedad fueron también más bajas, esto está en desacuerdo con lo mostrado por Leach y Garber (1970), quienes encontraron que al recudirse la infección se produjo un aumento en el rendimiento; pero concuerda con ellos en que las dosis altas del fungicida pueden causar fitotoxicidad, entorpeciendo el desarrollo y producción de las plantas. Bruggen (1986) mostró que la severidad de la enfermedad fue la única medida de la enfermedad que fue correlacionada negativamente conel rendimiento. El más alto rendimiento fue obtenido con la dosis de $6.81 \mathrm{~kg} / \mathrm{ha}$, pero este valor fue solamente $3 \%$ mayor que el testigo y $12 \%$ mayor que el rendimiento que se produjo en la dosis más alta de PCNB.

En el peso de 1,000 semillas se produjeron diferencias estadísticas para variedades, esto posiblemente es debido a los diferentes tamaños de semilla que posee cada variedad de las usadas en el trabajo, lo cual es un carácter genético. Sin embargo, el peso de las semillas no varió con el tratamiento químico.

\section{CONCLUSIONES}

El patógeno aún estando presente no afectó el rendimiento del fríjol.

El PCNB tuvo efecto profundo sobre todos los factores estudiados, pero el efecto fue aparentemente transitorio en los primeros estadios de la planta y este efecto negativo sobre el patógeno no influyó en un mayor rendimiento.

Revolución 84, aunque fue la variedad que registró la mayor severidad e incidencia de la enfermedad, no fue afectada en su producción de materia seca, ni en su rendimiento, ya que produjo el más alto valor.

Los resultados mostraron que la dosis de $6.81 \mathrm{~kg}$ /ha de PCNB fue la mejor en este trabajo, pero sólo aumentó el rendimiento en un $3 \%$ en comparación con el control y en un $12 \%$ en relación al resultado obtenido en el nivel de $11.36 \mathrm{~kg} / \mathrm{ha}$ del fungicida.

La dosis de $11.35 \mathrm{~kg} /$ ha aunque actuó positivamente contra la severidad e incidencia de la enfermedad, mostró cierto grado de fitotoxicidad que influyó negativamente en el crecimiento, desarrollo y producción de las plantas tratadas con el mencionado nivel de fungicida.

La mezcla de PCNB + Metalaxy1 no aumentó el rendimiento $\mathrm{M}$ frijol. Hubo una disminución de $3 \%$ en relación con el control.

\section{BIBLIOGRAFIA}

ABAWI, G.S. 1986. Root diseases of beans and their control strategies. Seminarios Internos, CIAT, Cali, Colombia.

ANDERSON, N.A. 1977. Evaluation of the Rhizoctonia complex in relation to seedling blight of flax. Plant Dis. Reptr. $61: 140-142$.

1982. The genetic and pathology of Rhizoctonia solani. Ann. Rev. Phytopathol. 20:329-347.

ARMDT, C.H. 1953. Evaluation of fungicides protectants of cotton seedlings from infection by Rhizoctonia solani. Plant Dis. ReptT. 37: 397-399.

BAKER,K.F. 1970. Types of Rhizoctonia diseases and their occurrence in: Rhizoctonia solani, Biology and Path, H.F. 1981. Root tot resistence in common bean germplasm of Latin America origen. Plant Dis. 65:485-489. 
BRISTOW, P.R.; KATAN, J. 1970. Protection from Rhizoctonia solani by PCNB acumulated in plants. (Abstract). Phythopethology 60: 1256.

; LOCKWOOD, J.L 1973. Control of Rhizoctonia solani by Pentachloronitrobenzene Accumulated from soil by bean plants. Phytopathology 63:808-813.

BRUGGEN, A. H.C.; WHALEN, C.H.; ANESON, P.A. 1986. Effects of inoculum level of Rhizoctonia solani an emergence, plant develo ment, and yield of dry beans. phytopathology $76: 869-873$

CARDOSO, J.E.-, ECHANDI, E. 1987. Biological control of Rhizoctonia root rot of snap bean with binucleate Rhizoctonia - like fungi. Plant Disease. 71:167-170.

DEKKER, J.; GEORGOPOULOS, S.G. 1982. Fungicide resistance in crop protection. Center of Agricultural Publishing and Documentation, Wageningen, Holland. p. 164, 168, 170.

GEBER, U. 1987. Occurrence of soil home pathogens on beans (Phaseolus vulgaris L.) in Nicaragua. Swedish University of Agricultural Sciences, Research Information Center. Uppsala, Sweden. $16 \mathrm{p}$.

GIBSON, I.A.S. 1961. An anomalous effect of PCNB on the incidence of damping-off caused by a Phytium sp. Phytopathology $51: 531-533$

KATAN, J.; LOCKWOOD, J.L 1970. Effect of Pentachloronitrobenzene on colonization of alfalfa residues by fungi and Streptomyces in soil Phytopathology 60:1578-1582.

KO, W.H.; ODA, M.K. 1972. The nature of control of Rhizoctonia solani by Pentachloronitrobenzene in soil. Phytopathology 62:385-387.

LEACH. LD.; GARBER, R.H. 1970. Control of Rhizoctonia solani in Rhizoctonia solani. Biology and Pathology. LR. Parmeter, Jr. ed. University of California Press, Berkeley. 255 P.

LIFSHTZ, R.; LIFSHTZ, S.; BAKER, R. 1985. Decrease in incidence of Rhizoctonia preemergence damping-off by use of integrated chemical and biological controls. Plant Disease 69:431434.

LLANO, G.A. 1997. Effects of fungicides an soil borne diseases on common bean (Phaseolus vulgaris L) in Nicaragua. Swedish 1. Agric. Res. 17-69-75.
; CAMPOS DE, C. 1983. Enfermedades del frijol común. In Manual de Producción de frijol común. Tapia, H. \& García, J.E. Edits. MIDINRA, Managua, Nicaragua. 200 p.

MARTIN, H. 1968. Pesticide Manual. Basic information on the chemicals used as active components of pesticides. British Crop Protection Council (BCPC), England.

McCARTER, S.M.; BARKSDALE. T.H. 1977. Effectiveness of selected chemicals in controlling Rhizoctonia fruit rot of tomato in greenhouse avid field tem. Plant Dis. Reptr. 61:811-815.

MOORE. W.D.; CONOVER, R-A. 1955. Chemicals soils treatments for the control of Rhizoctonia on snap bean. Plant Dis. Reptr. 39:103-105.

OGOSHI, A. 1997. Ecology and patogenicity of anastomogis and intraespecific groups of Rhizoctonia solani Kuhn. Arm. Rev. Phytopathology 25:125-143.

PARMETER, J.R. Jr.; SHERWOOD, R.T.; PLAIT W.D. 1969. Anastomosis grouping among isolates of Thanatephorus cucumeris. Phytopathology 59:1270-1278.

PASTOR - CORRALES, M. 1985. Enfermedades del frijol causadas por hongos. In Frijol investigación y producción. López, M. edit. CIAT, Cali, Colombia. 169-206 p.

RODRIGUEZ - KABANA, R.; CURL, E.A. 1980. Nontarget effects of pesucides on soilbome pathogens and disease. Am. Rev. Phytopathol. 18:311-332.

SCHWART7,H.F.;GALVEZ, G. 1980. Bean production problems. Disease, insect, soil, aid climatic constraints of Phaseolus vulgaris, CIAT, Cali, Colombia.

SHLEVIN, E.; KATAN, J. Rhizoctonia disease of carrot seedling and its control. Plant Dis. Reptr. 59:29-32.

STOCKWEI.1, V.; HANDLEY, P. 1987. Lignification of lesion border in Rhizoctonia - infected bean hypocotyls. Phytopathology 77: 589593

TAPIA, H.; GARCIA. J.E. 1983. Manual de producción de frijol común. MIDINRA, Managua, Nicaragua. 200 p.

WEBER, G.F. 1939. Web blight a disease of beans caused by Corticium microsclerotia. Phytopathology 29:559-575.

WORTHING, C.R. 1979. The pesticide manual. A World Compendium British Crop Protection Council. England. 